\title{
Cryogenic methods for biological small-angle x-ray scattering
}

\author{
D Moreau ${ }^{1}, \mathbf{R}$ Thorne ${ }^{2}$ \\ ${ }^{1}$ Cornell University, Ithaca, NY, ${ }^{2}$ Physics Dept, Cornell Univ. \\ dwm265@cornell.edu
}

Small-angle X-ray scattering (SAXS) is a key tool for probing the structure and function of proteins, nucleic acids, and macromolecular complexes. Most synchrotron sources have BioSAXS beam lines, but efforts to improve their throughput have not kept pace with user demand. Large sample volumes and low duty cycles are critical bottlenecks in the expansion of BioSAXS. The reduction in radiation damage at $\mathrm{T}=100 \mathrm{~K}$ significantly reduces the amount of protein required per measurement and sample holders compatible with standard macromolecular cryocrystallography infrastructure could allow for sample preparation in the home lab immediately after purification, easy sample storage and shipping, and automated high-throughput data collection. This will enable dramatically more efficient use of both biomolecules and synchrotron beam time, and significantly expand the potential scope of BioSAXS studies. Demonstrations of CryoSAXS have shown the potential of this technique1,2, however, the lack of a robust experimental platform has prevented CryoSAXS from becoming a routine method. The need to subtract a highly matched background scattering pattern from the macromolecule's scatter and the difficulty in vitrifying bulk-like solutions have posed serious technical challenges for the development of sample holders adequate for routine use. We have developed a new generation of CryoSAXS devices that have demonstrated encouraging results in synchrotron applications. These microfabricated devices have fixed pathlengths, low background scatter X-ray windows and small thermal mass, allowing for rapid vitrification. Each individual device is compatible with macromolecular cryocrystallography goniometer bases and pucks and allows for multiple samples per device. 1. Meisburger, S. P., et al., (2013) Biophys. J., 104, 227-236. 2. Hopkins, J. B., et al., (2015) J. Appl. Cryst. 48, 227-237.

Acta Cryst. (2020). A76, a42 